\title{
Investigating the Role of an Overview Device in Multi-Device Collaboration
}

\author{
Frederik Brudy ${ }^{1}$, Joshua Kevin Budiman ${ }^{1}$, Steven Houben ${ }^{2}$, Nicolai Marquardt ${ }^{1}$
}

${ }^{1}$ University College London, London, UK; ${ }^{2}$ Lancaster University, Lancaster, UK

f.brudy@cs.ucl.ac.uk, s.houben@lancaster.ac.uk, \{joshua.budiman.15,n.marquardt\}@ucl.ac.uk
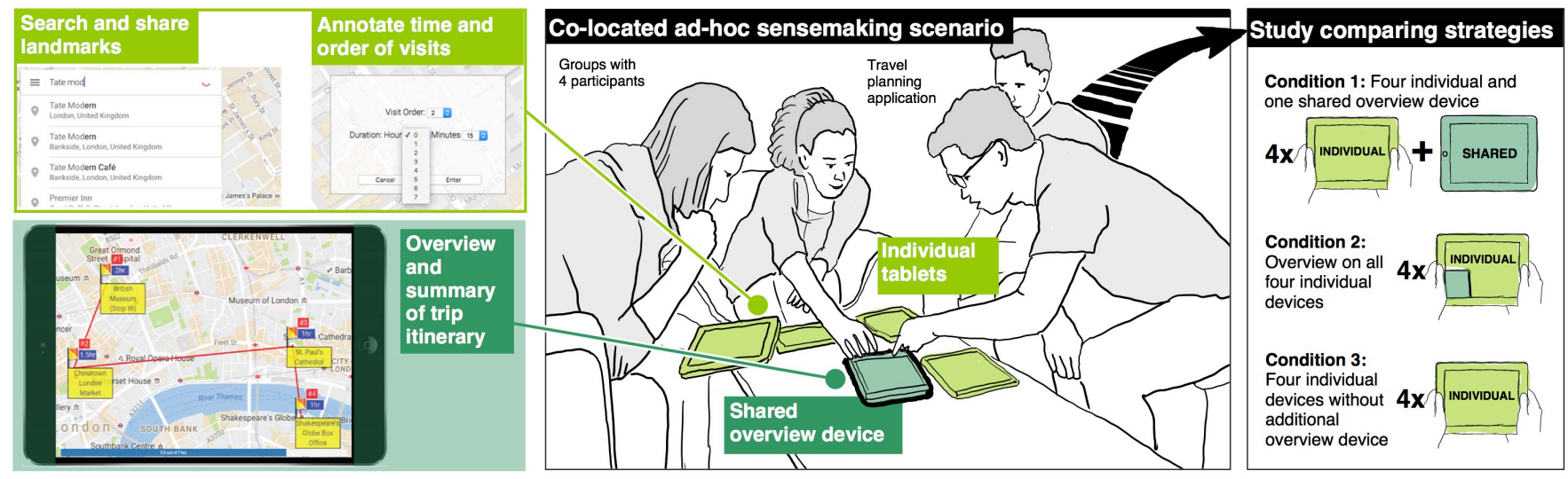

Figure 1. Using a collaborative travel planning application scenario (left), we study the effect of device configurations in a colocated multi-surface setting (centre) with three different conditions (right).

\begin{abstract}
The availability of mobile device ecologies enables new types of ad-hoc co-located decision-making and sensemaking practices in which people find, collect, discuss, and share information. However, little is known about what kind of device configurations are suitable for these types of tasks. This paper contributes new insights into how people use configurations of devices for one representative example task: collaborative co-located trip-planning. We present an empirical study that explores and compares three strategies to use multiple devices: no-overview, overview on own device, and a separate overview device. The results show that the overview facilitated decision- and sensemaking during a collaborative trip-planning task by aiding groups to iterate their itinerary, organize locations and timings efficiently, and discover new insights. Groups shared and discussed more opinions, resulting in more democratic decision-making. Groups provided with a separate overview device engaged more frequently and spent more time in closely-coupled collaboration.
\end{abstract}

Permission to make digital or hard copies of all or part of this work for personal or classroom use is granted without fee provided that copies are not made or distributed for profit or commercial advantage and that copies bear this notice and the full citation on the first page. Copyrights for components of this work owned by others than the author(s) must be honored. Abstracting with credit is permitted. To copy otherwise, or republish, to post on servers or to redistribute to lists, requires prior specific permission and/or a fee. Request permissions from Permissions@acm.org.

CHI 2018, April 21-26, 2018, Montreal, QC, Canada

(C) 2018 Copyright is held by the owner/author(s). Publication rights licensed to ACM.

ACM 978-1-4503-5620-6/18/04 ..\$15.00

https://doi.org/10.1145/3173574.3173874

\section{Author Keywords}

Multi-device interaction; decision-making; mobiles and tablets; ad-hoc collaboration; co-located collaboration

\section{ACM Classification Keywords}

H.5.3. Group and Organization Interfaces: Computer-supported cooperative work

\section{INTRODUCTION}

With the increased availability of mobile internet-connected devices, there is a shift in how people use information. The mobility of computing power has led to a nomadic workflow, in which people can access and process information 'on-thego'. People pull out devices at any point in time to access, share, filter through, and sort information. Technology helps people make sense of the increasing amount of information and ultimately influences people's decision-making process.

In general, such sensemaking activities are concernced with analysing information in a structured way by collaboratively collecting and organizing pieces of information to derive new knowledge, insights, and eventually arrive at the right result (i.e. knowledge product) [31]. In our work, we focus on the practices during a collaborative decision-making activity in an iterative process of foraging and sensemaking: a trip-planning task. Compared to a structured sensemaking task, in a collaborative decision-making task, there is not always a right or wrong answer and the decisions are influenced by the opinions people hold or the preferences they have [7]. Sharing information and opinions between individuals is important to yield a result that satisfies all those involved [39]. When a group fails to share information (e.g. through a lack of collaboration), there is an increased possibility of making a decision which does not satisfy everyone 
or even might be wrong $[7,39,46]$. Previous work found that group performance benefits when collaborators share and discuss task materials when engaging in closely-coupled collaborations [7,16,45]. Furthermore, the closeness of teams' collaboration and communication positively influences task performance and outcome [25]. Large interactive surfaces $[29,40,45]$ and combinations of mobiles can support collaborative work and help mediate ad hoc collaborations and decision-making activities [7,27,48]. A combination of mobile devices and a shared tabletop was found to lead to increased and better group collaboration [38]. However, meetings often happen spontaneously and the tedious setup of tabletop or wall displays contradicts the ad-hoc nature of meetings [36] - thus, previous work proposed to use mobile, personal devices in collaborative scenarios (e.g. [34,36]).

In this paper, we study how a group of people collaboratively plan a day-trip through London in such a multi-device bringyour-own-device (BYOD) ecology (Figure 1). A collaborative trip-planning task is an example of an iterative process of foraging and sensemaking, understanding the progress, and making decisions based on personal preferences, possibilities, and prior knowledge. We investigate if providing collaborators in a multi-device ecology with a shared space (an overview device) to organize their travel itinerary influences their sensemaking and decision-making practices. Our empirical study compares the practices in three conditions: (i) one collaborator brings an additional device that acts as a shared, always-on device, showing a summary of the progress; (ii) the overview is shown on each collaborator's device, but no additional shared device is available; (iii) each participant has only their own device and no overview is shown on each individual's device.

Our contribution is a study of how multiple tablets are used in ad-hoc decision-making activities. We observed that a separate overview device, with basic cross-device functionality, increases collaboration and leads to better decisionmaking when compared to having only personal tablets. Trips created by groups provided with the overview consisted of a more thought-through itinerary due to the groups doing multiple iterations of their work, accounting for more factors, spotting errors in their plans, and overall leading to a more democratic decision-making process. Those groups also perceived mental demand as slightly lower. Compared to having the overview on everyone's tablet, the use of an additional shared device resulted in more closely-coupled collaborations and more face-to-face discussions. Our study is the first to demonstrate that cross-tablet interfaces can successfully support collaborative decision-making whilst supporting advanced practices previously only observed in complex tabletop/wall-display setups (e.g. [23,25,44]).

\section{RELATED WORK}

Our research draws on the foundations of previous work on co-located collaborative sensemaking and multi-device support for collaboration.

\section{Collaborative decision-making and sensemaking}

Sensemaking allows a group to derive new insights through an iterative process [31] of foraging and sensemaking [43] where materials are collected and organized to interpret new information and to gain new insights [35]. Group collaborations have been shown to be beneficial for sensemaking, as a group can leverage each other's cognition [17,19,30,47] and collaborators can create a shared understanding of the available information [20]. Vogt et al. [43] reported that collaborators may take on two primary roles of foragers (gathers information and maintains an overall awareness of the state of the task) and sensemakers (dominant participant, who asks foragers to find documents, writes down key information, and directs the group). These roles are fluid and collaborators may switch roles throughout the process [43]. Isenberg et al. [25] observed several communication styles within tabletop collaborations. During a sensemaking task, participants in closely-coupled collaborations engaged in active face-toface communication. The ability to share and discuss relationships between task materials, whilst closely-coupled, benefits group performance [16,25]. In contrast, participants in loosely-coupled collaborations worked in parallel rather than together and focused more on their own task at hand with limited interaction. They were less successful in connecting key facts and completing their assigned task [25].

Previous work found that giving participants visual feedback of their participation in a group task on a shared display influenced participation by levelling out over- and under-participation [7,41] and that activity awareness benefits groups' performance in a remote collaboration [3]. In our study, groups were co-located and provided with an overview of the current task outcome, shown either on users' individual devices or on a shared device. It offers a go-to place for individuals to initiate a group discussion.

\section{Shared Spaces for Collaboration in Multi-Device and Large Screen Ecologies}

Previous research explored that an increased display size [1], multiple shared displays [33], and device ecologies [24] help a single user during sensemaking. Within collaborative situations, tabletops (e.g. [29,40]), wall projections (e.g. [6]), portable devices (e.g. $[15,48]$ ), or a combination thereof (e.g. $[2,6,21,22,45])$ can mediate collaborative sensemaking activities. In particular, such setups can provide a shared display $[6,33,45]$ and collaboration space $[2,40]$, support spatial and visual organization of content [49], provide awareness $[9,43]$, and support a shared understanding [22,45]. However, recent studies suggested that an increased size of a shared space is not indicative of improved collaborations, as participants' attention may be diverted towards the screen instead of other collaborators [49], and that users are hesitant to use multiple tablets in parallel, hinting at a "legacy bias" to use tablets as computers rather than documents [32].

In small group collaborations, using individual tablets negatively affects communications and engagement, when compared to using pen and paper [14]. Combining a shared tabletop with tablet devices helps to organize relevant materials 
on the shared space, whilst being able to work on individual tablets [45]. Providing a group, working on a tabletop, with a shared space to display an overview of results can facilitate co-located collaboration [25], and support group collaboration through mutual awareness and observability of users' actions [23]. Displaying different types of information on the shared display impacts a group's performance (i.e. a shared task overview facilitates monitoring group progress, whilst displaying replicated content facilitates conversational grounding) [44]. Using a wall display as a shared, physical reference point supports synchronization of group activity via body language and gaze [44].

However, although the benefits of a shared space on tabletop and wall displays have been shown, they are difficult to set up and do not easily support ad-hoc meetings, which contrasts to meetings often happening spontaneously [36]. How can we support ad-hoc collaboration tasks in a co-located setting, whilst still incorporating shared space? The related work of practices in a shared interaction space motivated our investigation of a shared display in a BYOD ecology.

\section{Multi-device Environments for Individual and Co-Located Sensemaking}

United Slates [5] and Conductor [15] enable a single user to use multiple tablets for cross-device sensemaking. In collaborative scenarios, MindMap [27] allow a co-located group to use mobile phones for collaborative brainstorming. HuddleLamp [34] creates spatial awareness across devices, only requiring a web browser on the client side. The design and implementation of these systems demonstrate the plausibility of using one's own personal devices to conduct collaborations. With Pass-Them-Around [26], a phone-based photo sharing application has been presented. MobiSurf [38] found that the combined use of personal and shared devices (with an interactive tabletop) facilitates switching between individual and group work activities. We extend this earlier work by investigating the benefit of an additional overview device vs. on-screen visual overview to support group activities.

\section{VOYAGEUR - COLLABORATIVE TRIP PLANNING}

To study co-located, collaborative trip planning in an ad-hoc BYOD setting, we implemented Voyageur, a multi-device collaborative web-based tool for planning a sightseeing tour through a city (Figure 1). Voyageur allows users to combine their personal devices, such as smartphones or tablets, into one interaction space, allowing them to create a shared itinerary. Each user can suggest and edit the group's shared map on their personal device whilst a summary of the trip is either shown on individual devices or on a separate overview device. Voyageur is a representative of a system that prior works have proposed in the cross-device domain for using personal devices to collaborate (e.g. $[5,15,27,34]$ ).

\section{Voyageur Implementation and Interaction}

Voyageur consists of a web application that allows one to search for locations, using a Google Maps interface. Locations can be shared with other users. Shared locations can be annotated with a visiting order and duration, thus creating a shared itinerary. A summary is provided as a zoomed-out map overview, where locations are connected according to their visiting order. A progress bar at the bottom of the screen shows how much time has already been allocated or whether a given time limit has been reached for the duration of the overall trip (in our study: 7 hours). In order to support ad-hoc collaborative sessions, we implemented Voyageur with the Connichiwa [36] web framework, which facilitates the distribution of web applications across multiple co-located devices.

Voyageur provides two different views (shown in Figure 1 and 2): overview (shown on the master or in an inset window on the client) and client (shown on any connected client). Both views show a geographical map (using Google Maps). The client application allows users to search for points-ofinterest using a text input. Whilst typing, search suggestions are presented and upon selecting one of the suggestions, a marker pin is dropped on the map. By touching the marker, the location can either be shared with the other users or deleted. Shared location markers appear on all connected devices and can be annotated with a visiting order and the duration (in hours and minutes) to add it to the shared trip itinerary. Annotations are synchronized across all devices. When shared markers are deleted, they are deleted on all devices. A summary is provided as an overview, where locations are connected per their visiting order, visualizing the air-path of the tour (Figure 1 left; Figure 2 right). A progress bar at the bottom of the screen shows the trip duration and remaining time of a given time limit (in our study: 7 hours).

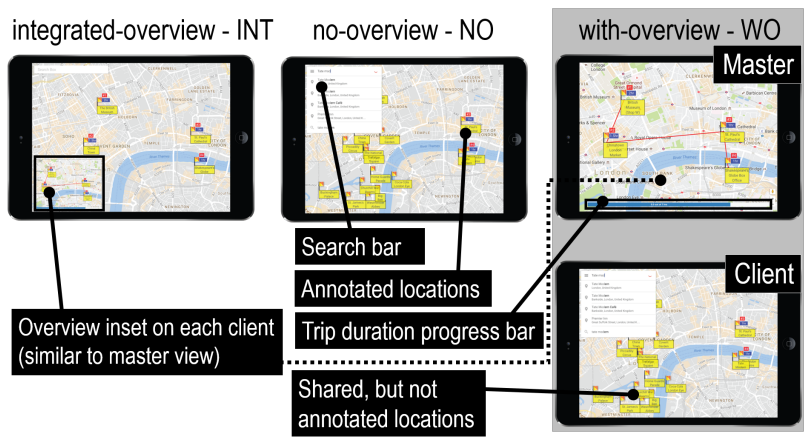

Figure 2. Comparison of the layouts for the study conditions.

Voyageur supports the following sense- and decision-making tasks as part of a collaborative trip-planning activity:

1) Facilitate sensemaking and decision-making. The visual overview of Voyageur allows one to detect patterns and derive insights about what to include in a trip. By providing all group members with access to the overview, Voyageur creates a shared understanding of the task, thus, assisting sensemaking [31] and decision-making [39]. It provides feedback about the current state and what has been accomplished.

2) Encourage closely-coupled collaborations. Closely-coupled collaborations foster more insights and higher task completion satisfaction $[25,40,44]$. Voyageur encourages closely-coupled collaborations by giving groups a shared always-on device, showing an overview of the progress. 
3) Encourage group discussion. Whilst increasing the amount of time people speak in a collaborative decisionmaking task is not directly congruent to their influence on the decision, an imbalance in participation can indicate that not enough details are being shared and not all participants' relevant viewpoints are discussed [7]. Voyageur enables group synchronization as well as spatial and activity awareness of explicit and implicit gestures through equal access of all users to the overview, thus, encouraging initiation of group discussions about items shown $[3,44]$.

4) Accommodate different roles. The roles of foragers and sensemakers for sensemaking tasks have been established [43], and it was pointed out that in a group scenario, collaborators frequently switch between these roles [25]. Voyageur allows for users to switch roles at any time by creating a shared understanding of current progress and equal access to information, thus, enabling flexibility of roles. Displaying the overview on a separate device allows users to touch or move the shared device. Encouraging explicit gestures, we aim to support implicit understandable roles [44]

\section{Sensemaking Tasks for Trip Planning}

Voyageur supports collaborative planning of a trip itinerary. Numerous applications have been developed to support individual users (e.g. Citymapper, Google Maps) and groups (e.g. Travefy, Triporam) in trip planning. However, these tools do not specifically support co-located collaboration. Previous research explored, for example, travel suggestions through geo-tagged photos [28] and calculating the best trip based on aggregated trip distance [18]. However, in an everyday scenario, people often use the combined interests and local knowledge of a group to decide on what to include in a trip and they might sit together to plan their itinerary, using their individual devices to find locations and information.

\section{STUDY}

Using Voyageur, we studied a collaborative trip planning activity supported by a separate overview device, whilst each person is using an individual tablet device. Through the study we wanted to learn if and how Voyageur (FP1) facilitates collaborative decision- and sensemaking practices during a co-located trip planning activity; (FP2) encourages closely coupled collaboration, thus fostering more insights and higher task completion satisfaction [25,40]; (FP3) encourages more sharing of information, thus enabling more discussions and consideration of more people's opinions [7]; (FP4) supports different roles of foragers and sensemakers [43] and allows for flexible transition between roles; and (FP5) accommodates different collaborative strategies as observed previously [25] (we will refer back to the focus points FP1-FP5 when reporting on the main findings in Listing 1). The task given to the group was to 'plan a day-tour through London', visiting important landmarks, lasting 7 hours.

\section{Participants}

We recruited 40 participants (21 female, 19 male) in groups of 4 from the local university and via snowball sampling. The only requirements were that (1) participants were familiar with London, having lived there for at least six months, and
(2) participants in each group knew each other. The second requirement was directly imposed by our task motivation (groups of strangers would not plan a day-trip through London together) and allowed participants a more relaxed communication setting. We recruited 22 students (postgraduate and $\mathrm{PhD}$ ) and 18 professionals (consultant, PA, teacher, etc.), aged between 23 and 37 years (Mean $(M)=30$ years; Standard Deviation $(\mathrm{SD})=4.1)$. All participants owned at least one touch device (smartphone, tablet, or smartwatch) and all but three reported that they used it often/daily. Two thirds $(67.5 \%)$ of participants owned two or more touch-enabled devices ( $M=1.9$; SD 0.7). All participants but one had prior experience in planning a trip as a group, primarily using Google Docs and Maps to collect links (e.g. accommodation, transportation, tourist websites, etc.) and their itinerary. Participants mentioned they use websites, guidebooks, travel apps, and their own or friends' local knowledge to plan things to do. They described their collaborative planning taking place in asynchronous remote settings (e.g. via email or text messages) as well as in co-located synchronous settings (e.g. at a friend's house or in a coffee shop).

F1: Overview allowed groups to detect patterns of locations that can be easily visited together (FP1).

F2: The overview created a shared understanding. Groups with an overview (WO, INT) tried out different options for timings and visiting order. This led to consideration of more options (FP3).

F3: WO and INT groups shared more opinions and more people shared their opinion (FP3). This resulted in a more democratic decisionmaking process (FP4, FP5).

F4: Overview led to iterations of the itinerary: This resulted in groups to spot and correct errors and allowed them to account for additional factors, such as lunch breaks and travel times (FP1, FP3).

F5: Displaying on separate device (WO) led to more closely-coupled collaboration and more face-to-face interactions by giving them a spatial and contextual focus point (FP2). It enabled group members to initiate a discussion spatially around the shared device.

F6: Territorial behavior: individual devices were rarely shared and only small glimpses occurred to them. The overview device was used equally by all group members.

Listing 1: Main findings (F1-F6) and their connection to the 5 focus points (FP1-FP5) of our study.

\section{Study Design}

To understand the influence of displaying Voyageur's overview on an additional device on group collaboration, we conducted a between-subject study, where we modified the presence and location of the overview: no-overview-device (NO), with-overview-device (WO), integrated-overview-on-individuals'-devices (INT) shown in Figure 2. In the INT condition, the overview was integrated in users' individual devices, and no additional device was given. In WO, the overview was displayed only on a separate device. No overview on individual devices and no additional overview device was given in the NO condition. We were interested in comparing the groups' practices and collaboration strategies emerging from the different device setups. We conducted our study in a between-subject design to (i) avoid bias through carry-over effect [13]; (ii) minimize range effects and avoiding contextual comparison of all tasks but the first task [13]; (iii) avoid experimenter demand effects and avoid drawing attention to 
the added/removed overview [4,13]; and (iv) avoid fatigue of participants due to study length and task repetition.

\section{Apparatus}

Participants were invited to our lab. After giving consent, they completed a pre-study questionnaire with basic demographic data, prior experience in collaborative travel planning, and device usage. Each participant was provided with an iPad Air 2 tablet with a 9.7" display; in the condition withoverview-device (WO) an additional iPad was provided. Participants were seated around a $118 \times 60 \mathrm{~cm}$ table (Figure 1 middle), with the researcher on the side as observer. Pen and paper were provided for completing the questionnaire and in case participants wanted to take notes during the study. After an introduction, participants were given a 5-minute training phase. After answering questions, each participant was given a separate subset of 5 out of the first top- 20 attractions in London [50] on a sheet of paper. Participants were then asked to plan a day out in London, exploring all or a subset of these attractions, lasting no longer than 7 hours. No time limit for task completion was given. Upon task completion, participants filled out a post-study questionnaire, rated task difficulty on a 10-point Likert scale (based on the NASA TLX), and a semi-structured interview was conducted. We conducted 4 sessions for WO, and 3 sessions for each NO and INT, each lasting 45-60 minutes (for a total of 10 sessions).

\section{Data Collection}

All sessions were video recorded to analyse groups' collaborative practices. In addition, notes were taken by the researchers during the study. Search input on each user's device, as well as actions to share, annotate and delete locations were logged by the system. We recorded touch input on the personal devices to quantify participants' interactions. "Touch activities" reflect the number of times the data log application captured tablets' touch coordinates, post-hoc grouped to a maximum of once every $50 \mathrm{~ms} /$ device. More touch activity therefore indicates busier interaction on the tablet due to increased touch movements from users (touchdown, -move, and -up). Questionnaires and semi-structured interviews were used to assess participant's prior knowledge and their intended strategies during their group work. The collected log data and video recordings were analysed using ChronoViz [8]. We coded the video for groups' collaboration practices, participants' interactions with each other, their device(s), and the focus of their interactions (e.g. being on a device, a collaborator, or content on the screen). The analysis of the materials collected during the study followed the grounded theory approach [10], using open coding of the video material and notes taken by the experimenter. We did not apply an existing coding scheme, but created codes as we encountered them, except for groups' collaboration styles (which we will discuss shortly). $40 \%$ of the videos were coded by two researchers, where the second researcher built upon the first's codes. The coding scheme was fully developed after $40 \%$. It consisted of the following categories: our 5 collaboration styles (Figure 3); gestures; conversation content; and miscoordination.

\section{RESULTS}

Our study showed that groups with an overview (INT and WO) went through multiple iterations of their trip itinerary whilst iterations were not observed in NO groups. Our findings indicate that overview groups reached decisions more democratically as more opinions were discussed. Displaying the overview on a separate device (WO) led to more closelycoupled collaborations and face-to-face interactions. Participants in INT/NO groups spent more time looking at their own devices, and less time in face-to-face interaction. The separate device gave participants a spatially stable anchor point to initiate closely-coupled collaboration and to base their shared interaction around. It provided group members a spatial and contextual focus point to meet other collaborators for discussions and sharing of information about items and the overall progress of their planned trip. In all conditions, we observed territorial behaviour with personal tablets [37], and pointing to, or touching someone else's device was rare. Listing 1 shows a summary of our main findings.

Three key themes emerged from our qualitative and quantitative analyses: (1) information organisation strategies, (2) applying the overview, and (3) collaboration styles.

\section{Theme 1: Information organisation strategies}

Our first theme describes how groups collect and organize locations to include in their itinerary.

\section{Collecting Locations}

Each group member was provided with a separate subset of five locations out of the top-20 attractions in London [50]. All ten groups created a subset of locations, as visiting all was not feasible because of the 7-hour time limit. We observed three approaches in creating subsets of the locations.

1) Listing all locations: Four groups (WO1, NO1, NO3, INT3 - see Table 1) shared all the locations with the other group members. Afterwards, participants engaged in group discussions to decide which location should be included in their itinerary. P38 suggested this approach explicitly to the group: "Shall we just all add our five locations and then see if we can already see where that leads us? I mean, maybe there's some of them close by [each other]".

2) Filtering locations before sharing: Two groups (WO4, INT1) spent the first minutes discussing the locations which they would like to include in the trip, without interacting much with the map (little to no touch activity). After this discussion, they then decided on the inclusion of each location in their trip, and only added it to the shared map if they decided to include it. Whilst creating their itinerary, they discussed their decisions in detail, and annotated each location after adding it. P29 described their process: "We started that each of us picked one location [as a] recommendation and we talked about it in the group and then decided together [whether to include it]. And only then added it to the [map]".

3) Mixed approach: Four groups (WO2, WO3, NO2, INT2) followed a mixed approach of these two strategies. Members first discussed general interests and which locations they had 
available, whilst not interacting with the map (little to no touch activity). After several minutes of discussion, they decided to share all 20 locations to identify places in walkingdistance to each other or that could be easily visited together.

\begin{tabular}{l|c|c|c|c|c}
\multicolumn{1}{c|}{ Group } & $\begin{array}{c}\text { Listing } \\
\text { all lo- } \\
\text { cations }\end{array}$ & $\begin{array}{c}\text { Filtering } \\
\text { locations }\end{array}$ & $\begin{array}{c}\text { Deleting/ } \\
\text { Clean-up }\end{array}$ & $\begin{array}{c}\text { Itera- } \\
\text { tion }\end{array}$ & $\begin{array}{c}\text { Identi- } \\
\text { fied } \\
\text { leader }\end{array}$ \\
\hline WO1 (P1-P4) & Yes & No & Yes & Yes & No \\
\hline WO2 (P5-P8) & Yes & Yes & No & Yes & Yes* \\
\hline WO3 (P9-P12) & Yes & Yes & Yes & Yes & No \\
\hline WO4 (P13-P16) & No & Yes & No** & Yes & No \\
\hline NO1 (P17-P20) & Yes & No & No & No & Yes \\
\hline NO2 (P21-P24) & Yes & Yes & No & No & Yes \\
\hline NO3 (P25-P28) & Yes & No & Some & No & Yes \\
\hline INT1 (P29-P32) & No & Yes & No** & Yes & No \\
\hline INT2 (P33-P36) & Yes & Yes & Yes & Yes & Yes* \\
\hline INT3 (P37-P40) & Yes & No & Yes & Yes & No
\end{tabular}

Table 1. The groups' activities. *=These groups' leader had an overall less dominant role compared to NO groups.

$* *=$ Because the group filtered their locations before sharing them, a cleanup was not needed.

\section{Organizing Locations}

After sharing locations, groups followed similar approaches to decide which places to include in their itinerary. All groups chose the visiting order based on estimates of shortest walking time, using their prior knowledge of the city or visually estimated walking distances (all groups were familiar with London). E.g., the first location to visit was often the closest to the starting point given by the study facilitator. P32 (INT1) said that the group then "went with the lowest common denominator [of interests]" to decide on places to visit.

Number and Timing of Annotating Locations: To finalize their itinerary, groups annotated the visiting order and duration of their locations. We observed greater average numbers of annotations in groups with overview (INT: $M=21.3$, $\mathrm{SD}=2.5$; WO: $\mathrm{M}=19.8, \mathrm{SD}=6.1$ ) than in the $\mathrm{NO}$ condition (NO: $\mathrm{M}=9.0, \mathrm{SD}=5.7$ ) - see Table 2. Groups with an overview (WO and INT) tended to annotate their visiting duration and order as they went along, thus they had frequent annotations distributed throughout the session, whereas NO groups only annotated very late towards the end of the session.

We suspected that a small number of annotations at a late stage of the process could be an indicator of a better thought through, and thus more stable, decision. Our video recordings however showed that NO groups were discussing their choices and changing their decisions in a similar way as WO and INT groups did, they just did not annotate it on the system. WO and INT groups were testing out different visiting duration and order as they went along. This was due to the overview being updated immediately and thus showing the status of the itinerary. They received immediate feedback on their annotations, enabling immediate evaluation of their decision. This often led to group discussions after new or updated annotations were shown on the overview. Groups without overview kept track of this information either in their head or on paper (only NO2 used paper). For NO groups, there was no benefit to annotating earlier in the process. However, this also resulted in fewer discussions about the consequences of an annotation and once a location was annotated, it was not discussed anymore (more about iteration in the next section).

Deleting and Clean-up Activities: Most groups in the WO and INT condition used the delete function to remove markers from the shared map after reaching the group's consensus whether they would like to visit the place. Three groups (WO2, NO1, NO2) did not delete any locations. WO4 and INT1 did not need to clean up, as they only had shared locations that they wanted to include. NO groups used the delete function only sparingly (Table 2).

\begin{tabular}{l|c|c|c|c} 
Cond. & Share & Annotate & Delete & Total \\
\hline WO & $\mathrm{M}=20.8, \mathrm{SD}=1.6$ & $\mathrm{M}=19.8, \mathrm{SD}=6.1$ & $\mathrm{M}=9.8, \mathrm{SD}=5.8$ & $\mathbf{M = 5 0 . 3}$ \\
\hline NO & $\mathrm{M}=17.0, \mathrm{SD}=5.7$ & $\mathrm{M}=9.0, \mathrm{SD}=5.7$ & $\mathrm{M}=1.3, \mathrm{SD}=1.2$ & $\mathbf{M = 2 7 . 3}$ \\
\hline INT & $\mathrm{M}=18.3, \mathrm{SD}=4.5$ & $\mathrm{M}=21.3, \mathrm{SD}=2.5$ & $\mathrm{M}=7.3, \mathrm{SD}=4.5$ & $\mathbf{M = 4 7 . 0}$
\end{tabular}

Table 2. Average number of executed commands per group in each condition and average total number of all commands.

\section{Theme 2 - Applying the Overview}

The overview allowed WO and INT groups to coordinate times, identify patterns, and gave groups a shared understanding about the current discussion. The overview encouraged these groups to iterate over their plan and refine visiting duration and order to account for more factors, such as opening times and lunch breaks. Displaying the overview on a separate device (WO) gave the groups a spatial anchor point to turn to and initiate discussions and collaboration, and coordinate their interaction with each other.

\section{Overview to Detect Patterns (F1)}

Most groups shared all 20 locations (Table 1) and then identified clusters of locations that are within short walking distances of each other. We observed that WO groups discussed whether each cluster should be included in the trip or not, whilst looking at it together on the shared device. They then decided to visit a subset of places from that cluster. INT and NO groups also employed the clusters to identify locations to be visited. However, the lack of the shared device made it more difficult to coordinate the discussion with group members: they had to verbally communicate what they were looking at, rather than pointing it out on the shared device. NO and INT groups frequently spent time navigating around their maps to coordinate what the group was discussing. Even though INT groups also had an overview integrated on individual devices, participants had to find the location being currently discussed by panning and zooming on their own maps. Only in a few occasions did participants turn their own device around, showing the others what they were looking at. The device was then mainly shown to immediate neighbours, resulting in smaller sub-group collaborations.

\section{Overview and Iteration (F4)}

After groups finished planning their 7-hour trip, WO1, INT1, and INT2 decided to iterate their plan, by one team member explicitly suggesting this iteration phase to identify errors in their annotations (e.g., ensuring they had planned lunch or to allow enough time for walking). Although the other WO and INT groups did not declare an iteration phase, the analysed 
video and touch data showed that the groups discussed each included location at least twice, showing patterns of iteration. The iteration allowed the groups to identify and correct errors, resulting in a more thoroughly defined and thoughtthrough trip, accounting for more factors. We found that NO groups did not iterate their plan: once groups had decided to include a location in the itinerary, they did not reconsider that decision. This observation was confirmed by the logged interactions with the system: all interactions with a particular location in NO were located around the time of their first annotation. Post-hoc changes to these annotations occurred rarely.

\section{Overview created a shared understanding (F2)}

We observed that for groups without the overview (NO; Table 1) a leader often kept an eye on the status and updated the group about progress. If the group did not reach a decision, the leader took charge and decided on the group's behalf. We also observed a leader in INT2 and WO2, but they were less dominant in this role and eventually these groups came to decisions in a similar way to all other WO and INT groups: by discussing their options and eventually making a group decision about points of interest, visiting duration, and order.

\section{Theme 3 - Collaboration Styles (F3, F5)}

In our analysis, we were interested in what patterns of collaborations participants in the different conditions adapted. We extended and re-appropriated the coupled collaboration styles, previously developed by Tang et al. [40] and Isenberg et al. [25], for a study with groups of four (rather than paired collaborations) and for a multi-device scenario. We focus on where attention was amongst team members (e.g., face-to-face or towards their device/s). Our collaboration styles consist of the following and are depicted in Figure 3:

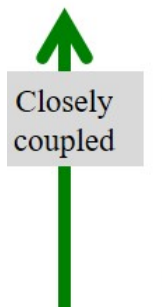

1. Active Discussion (AD). Face-to-face discussions amongst group members. Limited interactions with the system (e.g., pointing to screens or scrolling).

\section{Single-Shared View} (SSV). All group members allocate their attention to one single device.

3. Disjoint and Shared View (DSV). 2-3 members focus their attention to a single device whilst others continue discussions.

4. Disjoint and Distributed View (DDV). 1-2 group members are focusing on their own device, whilst others continue discussions.
5. Distributed View (DV). All group members are focusing on their own device. Minimal to no discussions.

\section{Shared Attention}

Our video data showed a trend for WO to engage in more SSV $(25 \%$ of their total time) than NO $(6 \%)$ or INT $(3 \%)$ (see Figure 7). In WO groups, we observed that this usually was initiated by a group member looking up from their own device and addressing the group by talking about content on the overview device (e.g. by pointing to it or verbally referring to it). Although no overview device was provided, SSV still occurred in NO and INT groups, usually only with two people sitting next to each other. These SSVs generally occurred when a group member started annotating a location, asking other members for their opinion, and then exchanging small glimpses at each other's tablets. However, occurrences of SSV in NO and INT groups tended to be less likely and shorter, as group members quickly reverted to their own tablet, resulting in DV or DDV. Since NO2 used pen and paper to keep track of timings, we observed some occurrences of SSV in this group around the paper. In our study, we observed that SSV led more frequently to active discussion (AD), without groups focusing on a device anymore. This mainly occurred when SSV was initiated by a group member raising a concern or asking a question. Participants then used the shared device to find common ground for their discussion. If the information on the device was then not needed anymore, they continued their discussion (Figure 4).

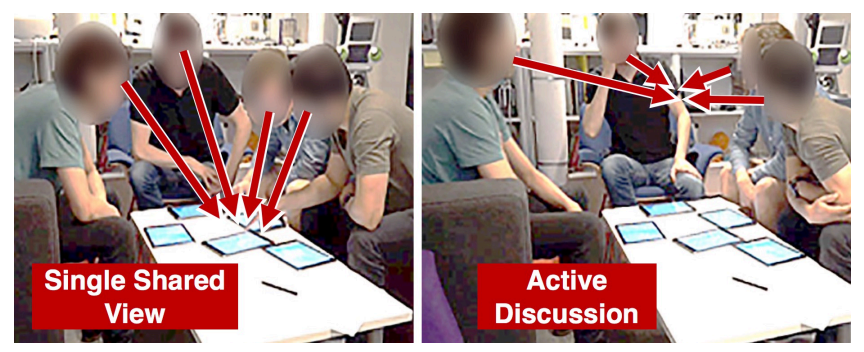

Figure 4. Shared attention on the overview device (SSV; left) often led to active discussion (AD; right) as the device gave the group a common focus and starting point for a diccussion.

\section{Pointing}

Our study indicates that pointing gesture could trigger shared attention. We observed fewer occurrences of pointing in $\mathrm{NO}$ and INT groups $(\mathrm{M}=7.88$ / group in $\mathrm{WO}$; $\mathrm{M}=2.7$ for $\mathrm{NO}$; $\mathrm{M}=3.3$ for INT). Members in WO group tended to point towards the overview device, which resulted in other members engaging in SSV or DSV. For instance, P8 in Figure 5 (left) points to the overview, prompting other members to move their attention towards the overview and start discussions about lunch. Figure 5 (right) shows how P29 pointed at her screen to discuss a location. Another participant briefly looked at the screen but quickly shifted her attention back to her own tablet. Other participants did not notice, as they were focussing on their own screens. In general, we observed that the overview device led to more shared attention and closelycoupled discussions by giving participants a shared focus space. 

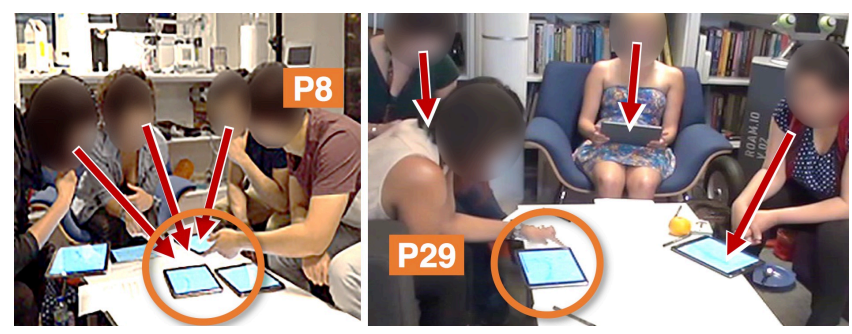

Figure 5. Left: P8 (WO2) points toward overview device, other members shifted their attention to it. In NO groups pointing

rarely led to shared attention (right): P29 (INT1) points toward her device; other members' focus stays on own devices.

\section{Distributed Attention}

For groups without the separate device we observed that they spent more than half their time looking at their own devices (DDV+DV; NO: 57\%; INT: 60\%; in contrast WO: 27\%). The data logs showed higher average touch activity per minute for groups without an overview device (NO: 424 / minute; INT: 226 / minute) than in WO groups (135/ minute). As a reminder: higher values for touch activity indicated more user-device interactions. When we analysed our video data, we noticed that NO/INT group members, who engaged in DV or DDV, were more likely to pan and zoom on their map to follow what other members were discussing. This aligns with the observation that NO groups were interacting more with their individual tablets. However, the increased touch activity in NO groups does not necessarily imply greater number of commands (share, annotate, delete) executed: WO and INT groups executed more commands on average per group (WO: $\mathrm{M}=50.3$ commands / group; INT: $\mathrm{M}=47.0)$, compared to $\mathrm{NO}$ groups $(\mathrm{M}=27.3)$ (Table 2).

\section{Active discussions}

Active discussions (AD) were present in all groups. We observed that $\mathrm{WO}$ groups not only initiated more $\mathrm{AD}$ on average per group (WO: $\mathrm{M}=13.0, \mathrm{SD}=3.24 ; \mathrm{NO}: \mathrm{M}=10.7$,
$\mathrm{SD}=3.09$; INT: $\mathrm{M}=11.0, \mathrm{SD}=2.94$; Figure 7), they also engaged in longer discussions ( $\mathrm{M}=30$ seconds, $\mathrm{SD}=10 \mathrm{~s})$ compared with NO $(M=20 \mathrm{~s}, \mathrm{SD}=4 \mathrm{~s})$ and INT $(\mathrm{M}=24 \mathrm{~s}, \mathrm{SD}=4 \mathrm{~s})$. The content of conversations in NO groups mostly consisted of a participant asking a question to confirm key facts or inquire about information, whilst other group members gave brief and passive responses. Longer discussions were focused around facts about locations someone was sharing or personal interests of group members. For example, in one instance of AD (WO1, duration 41s), P4 asked his collaborators for more information about what Horse Guards Parade is. The same question was also raised in NO1. However, in NO1 the AD ended quickly and turned into a DV, when the other members focused on their own devices trying to locate it. Ultimately, whilst doing so, one group member got sidetracked and asked a question about a different location.

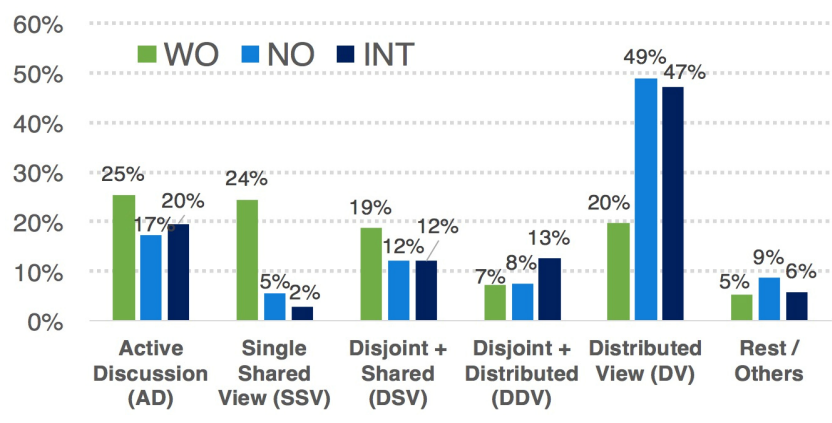

Figure 7. Percentage of time spent in the different kinds of collaboration, by condition.

We observed that the presence of the overview device gave group members the opportunity to direct their attention to a shared space. Though discussions may not involve face-toface interactions, group members still shared their attention onto the overview device and engaged in discussions centred on the same topic.

\section{Territorial behaviour (F6)}

In all groups, personal devices were
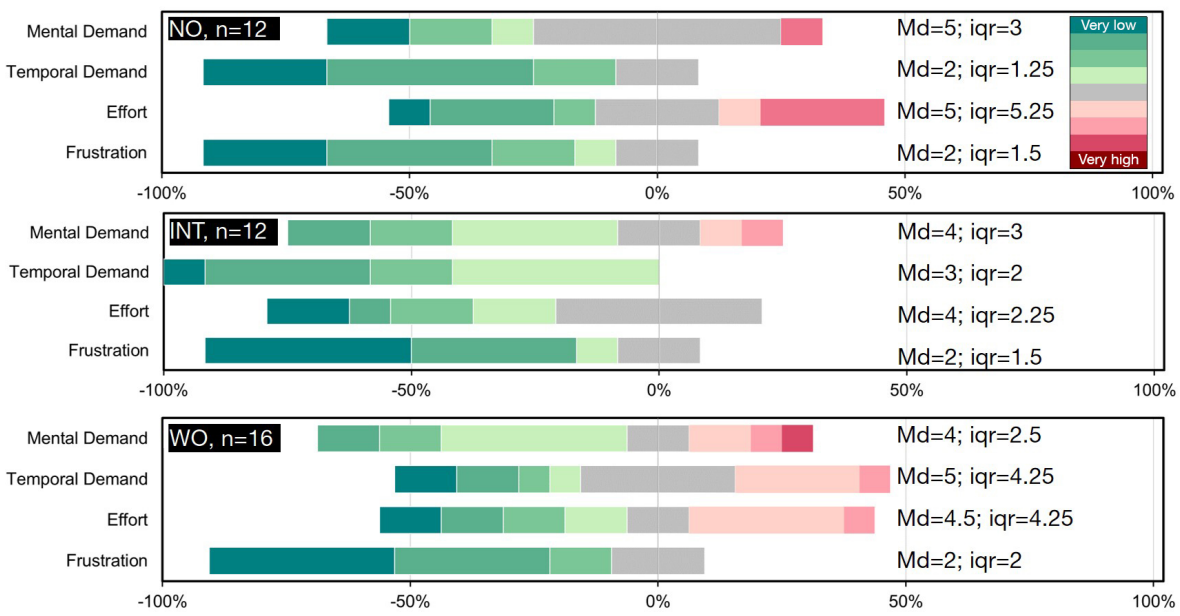

Figure 6. Results of answers to the post-study questionnaire, rating mental demand, temporal demand, effort, and frustration for groups without overview (NO), overview displayed on everyone's device (INT), and overview on separate device (WO). Likert scales from 1 (very low) to 10 (very high). shown to other people but there was never shared access to individuals' devices. All participants respected that others' devices were usually not for them to interact with. They rarely touched and interacted with someone else's device and if so, only after explicit consent. Although in two groups the overview device was picked up by participants (WO3 and WO4), this was only brief and the device was placed back into the shared space soon after.

\section{User Feedback}

Participants learned how to use the system well within the training phase. The shared itinerary helped to keep track of what other group members were doing. P29 stated that she "liked 
that [she] could see on [her] iPad what other people [did]" and P31 said the shared collective itinerary was "useful for tracking what others are doing".

In WO conditions, participants appreciated the overview device as useful for the group's discussion (e.g. P8 and P2). P5 stated that the "two screens were good. So, you have on one side the search [...] and on the other the overview", and further she "only used [her] own tablet when searching for a location. [When] discussing something [she] always used the overview". INT groups were presented with the same information on their own device, as WO groups on the separate device. P33 (INT2) said that it "would have been great to have the overview constantly on for example this TV here (points to TV). So, you see immediately the bigger picture and don 't have to zoom around [on your own tablet]". However, some issues arose through positioning, as P8 (WO2) reported: "For me [the overview device] was upside down. [I got] at least an idea, but could not read anything". Some participants asked for more information on who shared or annotated a location. For example, P31 said "when someone edited something you don't know who's doing it. So, if there's like a bubble saying who did it would be good". On the contrary, P16 stated, that he "like[d] that you don't see what someone else did. So, you can't be put on the spot".

Participants in the NO groups reported that they were missing a time-keeping feature and an easily visible summary of the visiting order or path - both components of the overview. The post study questionnaire showed slightly higher responses to the question 'how mentally demanding was the task' for the NO condition (Figure 6, mental demand; NO: Median $(\mathrm{Md})=5$, interquartile range (iqr) $=3$; INT: $M d=4$, $\mathrm{iqr}=3$; $\mathrm{WO}: \mathrm{Md}=4$, iqr=2.5). However, although this difference is only very small, our analysed video data indicated that this might have resulted from the NO groups having to keep track of the overview's information either in their head or on paper.

\section{DISCUSSION}

Previous research around tabletops has shown many benefits for collaborative decision- and sensemaking activities, such as shared understanding [22,45], mutual awareness [25], more closely-coupled collaboration and, thus, more sharing of information and discussions [16,25]. However, we increasingly encounter multi-user and multi-device scenarios, and research is shifting towards supporting such scenarios. Using individual devices has shown to negatively affect communication and engagement [14] and people are hesitant to use multiple tablet devices in parallel [32].

Guided by the question of how we can support ad-hoc decision- and sensemaking with a shared space, our study explored practices of using additional devices as a shared and flexible overview device in a collaborative co-located trip planning task. The results indicate that adding an overview with a summary of the trip itinerary facilitated sense- and decision-making. Similar to previous work around tabletop displays (e.g. $[16,25,45])$, we observed that groups with a shared overview tended to be more effective in their decision- and sensemaking. We observed that groups with a shared device worked in more closely-coupled collaborations, leading to more active discussions. These discussions were important for groups in their trip planning activity (similar to $[7,39])$. The overview tablet in our study was more successful compared to a previous study [32], likely because their study comprised only of dyads where everything a participant said was directed to the other. We observed however, that verbal discussions were not always directed to specific individuals, meaning some context could be lost. Similar to previous work we found that a shared display supported groups' synchronization through explicit and implicit gestures, spatial awareness of activities [44], as well as activity awareness [3].

\section{Space and Focus}

As a shared device that every member could engage with equally, we observed that the overview device (WO) was used as a spatial focus point: whenever someone initiated a discussion around a location, they could point to that location on the shared device, automatically shifting the group's attention towards the device through a clearly visible gesture. When pointing to one's own device (especially in NO and INT groups), drawing attention from other group members proved difficult, as they were engaged with their own device. Interacting with, or pointing towards someone else's device was rare and short-lived. Although in INT, some screen space was covered by the overview, participants did not comment negatively on lack of space for the "primary" interaction, nor could we observe any changed behaviour as a result of the covered space.

We observed that WO groups spent more time in discussions which were also more frequent. Groups without a separate device (NO, INT) focused more and for longer periods on their own devices. As group members were focused on their own screens, eye contact was less likely to develop, which discouraged potential active discussions and led to comments being ignored by the group. These findings, using mobile devices, are in line with previous findings around fixed tabletop displays, where groups were working in tighter coupled collaboration when working closely together [40].

Interestingly, only two of the WO groups moved the overview device (WO3, WO4), whilst the other groups treated it like a fixed display. In those groups that moved the overview device, we observed more spatial movement as participants were shifting on their chairs or standing up to have a better view (similar to as observed previously [32]). This spatial movement also seemed to contribute to more closely coupled collaborations as participants used the movement to point out locations on the shared device and initiate a discussion. Whether the more closely-coupled discussions were a result of the movement, or the movement was a result of more closely-coupled collaboration, needs further investigation.

The overview device also functioned as a contextual focus point, by providing a shared space to initiate discussions 
about displayed information. Previous work around tabletop displays found that collaborators prefer common, global views when in tighter coupled collaboration [40]. In our study, several discussions that started about items on the overview device turned into face-to-face conversations. WO groups' discussions tended to be more fruitful, communicating relationships between locations and personal preferences, and participants considered more factors such as opening times and lunch breaks. In contrast, we observed more distributed attention and short-lived conversations in NO and INT groups.

Although we observed shared views in all groups, this mostly meant shared viewing of someone's device. Participants only rarely shared access to devices. Since all information was shared with everyone else we expected participants in INT to more freely share their devices. However, similarly to prior work around tabletop displays [37], we observed that participants in a multi-device collaboration respected each other's territory and rarely interacted with someone else's device (and only after explicit invitation).

\section{Decision-Making and Sensemaking}

A decision-making task is strongly influenced by people's opinions [7], and therefore it is one important goal that group members have equal opportunities to share their thoughts and express their opinions. Through video analysis and the exit interviews we found that providing groups with an overview builds a shared understanding for group members and resulted in a more democratic decision-making. In WO and INT, members had equal access to the same information, and we observed that groups shared more opinions and made decisions where everyone's voice had been heard.

Neither NO group iteratively created their agenda. Giving groups an overview (WO, INT) we noticed iterations of the itinerary after groups finished a first draft. We observed that in WO groups, members collaboratively looked at the shared device, which in return led to more insights and ideas through discussion. This iterative process (i.e. [31]) tended to lead to more refined decisions and finding and correcting of mistakes (e.g. missing lunch breaks or travel times).

\section{Limitations and Future Work}

Voyageur was implemented for a simple collaborative trip planning task. We are currently extending our research to other areas of knowledge work, such as curation works (e.g. [2]). The current design requires participants to explicitly share their locations with the rest of the group. Previous research $[12,25]$ suggested that implicit sharing of information is beneficial for collaborative analysis. It will be interesting to understand how translucence [11] and feedforward [42] influence coordination and performance.

People now own multiple touch enabled devices, as our questionnaire data showed $(\mathrm{M}=1.9$; SD 0.7). Voyageur requires adding one more device than people to a configuration, resulting in the need for additional hardware and potentially added cost. Whilst other devices such as smartphones or laptops could also be used to display the overview, it remains for future work to investigate how other form factors influence a group's collaboration practices. Having an additional device might also limit the number of people collaborating, as issues such as reach and occlusion might arise.

We conducted our study as a between-subject design, to avoid bias through carry-over effects [13], minimize range effects [13], avoid experimenter demand effects [4,13], and avoid fatigue of participants. Conducting this study in a within-subject design is a possible alternative setup, but would require several changes in the study setup. First, a new set of locations has to be provided for every condition to avoid carry-over and range effects, though carefully chosen to have similar spatial and contextual properties to be able to observe equal secondary effects (e.g., cluster pattern detection and discussions about walking distance). Second, adding or removing a single aspect of the study (in our case the overview) would draw attention to this and might result in participants adapting their behaviour $[4,13]$. This requires careful consideration, not only through counterbalancing, but potentially through a 'decoy condition/item/task'. Third, the overall length of the task needs to be monitored. Since our interest was in the qualitative insights in groups' practices, the length of discussions and groups' sensemaking and decision-making processes are difficult to control.

\section{CONCLUSION}

In summary, with our study we found trends that the overview allowed users to detect patterns of locations (F1), led to consideration of more factors through a shared understanding (F2), and eventually allowed groups to iterate their itinerary (F4). Displaying the overview on a separate device encouraged more closely-coupled collaborations (F5) which resulted in sharing of more information and more active discussions. This in return led to a more democratic decisionmaking process (F3). Whilst we observed territorial behaviour with personal devices (F6), the overview device acted as a spatial and contextual focus point for collaborators to initiate discussions (F5) and focus their attention, and aided groups in mediating their collaboration.

Many previous works have proposed cross-device and multidevice interactions and systems for collaborative works, but only few have been studied in everyday practices. Whilst our study was conducted in the lab and with the focus on a specific activity - collaborative trip planning - we believe that the insights show merits for real-world collaborative multidevice settings. We showed how changing device configuration benefits groups' exchange of information and their collaboration during their sensemaking and decision-making activity. This is promising for future work on deploying multidevice systems in everyday situations.

\section{ACKNOWLEDGMENTS}

We thank anonymous reviewers for helpful feedback and all our study participants. This work has been supported by Microsoft Research through its PhD Scholarship Programme. 


\section{REFERENCES}

1. Christopher Andrews, Alex Endert, and Chris North. 2010. Space to think: large high-resolution displays for sensemaking. In Proceedings of the SIGCHI Conference on Human Factors in Computing Systems (CHI '10), 55-64. Retrieved July 19, 2015 from https://doi.org/10.1145/1753326.1753336

2. Frederik Brudy, Steven Houben, Nicolai Marquardt, and Yvonne Rogers. 2016. CurationSpace: Cross-Device Content Curation Using Instrumental Interaction. In Proceedings of the 2016 ACM on Interactive Surfaces and Spaces (ISS '16), 159-168. https://doi.org/10.1145/2992154.2992175

3. John M. Carroll, Dennis C. Neale, Philip L. Isenhour, Mary Beth Rosson, and D. Scott McCrickard. 2003. Notification and awareness: synchronizing task-oriented collaborative activity. International Journal of Human-Computer Studies 58, 5: 605-632. https://doi.org/10.1016/S1071-5819(03)00024-7

4. Gary Charness, Uri Gneezy, and Michael A. Kuhn. 2012. Experimental methods: Between-subject and within-subject design. Journal of Economic Behavior \& Organization $81,1: 1-8$. https://doi.org/10.1016/j.jebo.2011.08.009

5. Nicholas Chen, Francois Guimbretiere, and Abigail Sellen. 2012. Designing a multi-slate reading environment to support active reading activities. ACM Transactions on Computer-Human Interaction 19, 3: 1-35. https://doi.org/10.1145/2362364.2362366

6. C-W. Chung, C-C. Lee, and C-C. Liu. 2013. Investigating face-to-face peer interaction patterns in a collaborative Web discovery task: the benefits of a shared display. Journal of Computer Assisted Learning 29, 2: 188-206. https://doi.org/10.1111/j.13652729.2012.00493.x

7. Joan Morris DiMicco, Anna Pandolfo, and Walter Bender. 2004. Influencing Group Participation with a Shared Display. In Proceedings of the 2004 ACM Conference on Computer Supported Cooperative Work (CSCW'04), 614-623. https://doi.org/10.1145/1031607.1031713

8. Adam Fouse, Nadir Weibel, Edwin Hutchins, and James D. Hollan. 2011. ChronoViz: A System for Supporting Navigation of Time-coded Data. In CHI '11 Extended Abstracts on Human Factors in Computing Systems (CHI EA '11), 299-304.

https://doi.org/10.1145/1979742.1979706

9. Darren Gergle, Robert E. Kraut, and Susan R. Fussell. 2004. Language Efficiency and Visual Technology Minimizing Collaborative Effort with Visual Information. Journal of Language and Social Psychology 23, 4: 491-517. https://doi.org/10.1177/0261927X04269589
10. Barney G. Glaser and Anselm Strauss. 1967. The discovery of grounded theory: Strategies for qualitative research. Aldine Publishing, New York.

11. Nitesh Goyal and Susan R. Fussell. 2016. Effects of Sensemaking Translucence on Distributed Collaborative Analysis. In Proceedings of the 19th ACM Conference on Computer-Supported Cooperative Work \& Social Computing (CSCW' '16), 288-302. https://doi.org/10.1145/2818048.2820071

12. Nitesh Goyal, Gilly Leshed, Dan Cosley, and Susan R. Fussell. 2014. Effects of Implicit Sharing in Collaborative Analysis. In Proceedings of the SIGCHI Conference on Human Factors in Computing Systems (CHI '14), 129-138. https://doi.org/10.1145/2556288.2557229

13. Anthony G. Greenwald. 1976. Within-subjects designs: To use or not to use? Psychological Bulletin 83, 2: 314. Retrieved August 30, 2017 from http://psycnet.apa.org/journals/bul/83/2/314/

14. Jonathan Haber, Miguel A. Nacenta, and Sheelagh Carpendale. 2014. Paper vs. Tablets: The Effect of Document Media in Co-located Collaborative Work. In Proceedings of the 2014 International Working Conference on Advanced Visual Interfaces (AVI '14), 89-96. https://doi.org/10.1145/2598153.2598170

15. Peter Hamilton and Daniel J. Wigdor. 2014. Conductor: enabling and understanding cross-device interaction. In Proceedings of the SIGCHI conference on Human factors in computing systems (CHI '14), 27732782. Retrieved July 19, 2015 from http://dl.acm.org/citation.cfm?id=2557170

16. Preben Hansen and Kalervo Järvelin. 2005. Collaborative Information Retrieval in an information-intensive domain. Information Processing \& Management 41, 5: 1101-1119. https://doi.org/10.1016/j.ipm.2004.04.016

17. Richard Harper and Abigail Sellen. 1995. Collaborative Tools and the Practicalities of Professional Work at the International Monetary Fund. In Proceedings of the SIGCHI Conference on Human Factors in Computing Systems (CHI '95), 122-129. https://doi.org/10.1145/223904.223920

18. Tanzima Hashem, Sukarna Barua, Mohammed Eunus Ali, Lars Kulik, and Egemen Tanin. 2015. Efficient Computation of Trips with Friends and Families. In Proceedings of the 24th ACM International on Conference on Information and Knowledge Management (CIKM '15), 931-940. https://doi.org/10.1145/2806416.2806433

19. Jeffrey Heer and Maneesh Agrawala. 2008. Design Considerations for Collaborative Visual Analytics. Information Visualization 7, 1: 49-62. https://doi.org/10.1057/palgrave.ivs.9500167 
20. Morten Hertzum. 2008. Collaborative information seeking: The combined activity of information seeking and collaborative grounding. Information Processing \& Management 44, 2: 957-962. https://doi.org/10.1016/j.ipm.2007.03.007

21. Tobias Hesselmann, Niels Henze, and Susanne Boll. 2010. FlashLight: Optical Communication Between Mobile Phones and Interactive Tabletops. In ACM International Conference on Interactive Tabletops and Surfaces (ITS '10), 135-138. https://doi.org/10.1145/1936652.1936679

22. Otmar Hilliges, Lucia Terrenghi, Sebastian Boring, David Kim, Hendrik Richter, and Andreas Butz. 2007. Designing for collaborative creative problem solving. In Proceedings of the 6th ACM SIGCHI conference on Creativity \& cognition (C\&C), 137-146. Retrieved September 18, 2015 from http://dl.acm.org/citation.cfm?id $=1254980$

23. Eva Hornecker, Paul Marshall, Nick Sheep Dalton, and Yvonne Rogers. 2008. Collaboration and Interference: Awareness with Mice or Touch Input. In Proceedings of the 2008 ACM Conference on Computer Supported Cooperative Work (CSCW '08), 167-176. https://doi.org/10.1145/1460563.1460589

24. Steven Houben, Paolo Tell, and Jakob E. Bardram. 2014. ActivitySpace: Managing Device Ecologies in an Activity-Centric Configuration Space. In Proceedings of the Ninth ACM International Conference on Interactive Tabletops and Surfaces (ITS '14), 119128. https://doi.org/10.1145/2669485.2669493

25. Petra Isenberg, Danyel Fisher, Paul Sharoda A., Meredith Ringel Morris, Kori Inkpen, and Mary Czerwinski. 2012. Co-located Collaborative Visual Analytics Around a Tabletop Display. IEEE Transactions on Visualization and Computer Graphics 18, 5: 689-702. https://doi.org/http://dx.doi.org/10.1109/TVCG.2011.2 87

26. Andrés Lucero, Jussi Holopainen, and Tero Jokela. 2011. Pass-them-around: Collaborative Use of Mobile Phones for Photo Sharing. In Proceedings of the SIGCHI Conference on Human Factors in Computing Systems (CHI '11), 1787-1796. https://doi.org/10.1145/1978942.1979201

27. Andrés Lucero, Jaakko Keränen, and Hannu Korhonen. 2010. Collaborative Use of Mobile Phones for Brainstorming. In Proceedings of the 12th International Conference on Human Computer Interaction with Mobile Devices and Services (MobileHCI '10), 337-340. https://doi.org/10.1145/1851600.1851659

28. Abdul Majid, Ling Chen, Gencai Chen, Hamid Turab Mirza, and Ibrar Hussain. 2012. GoThere: Travel Suggestions Using Geotagged Photos. In Proceedings of the 21st International Conference on World Wide Web
(WWW'12 Companion), 577-578. https://doi.org/10.1145/2187980.2188135

29. Miguel A. Nacenta, Mikkel R. Jakobsen, Remy Dautriche, Uta Hinrichs, Marian Dörk, Jonathan Haber, and Sheelagh Carpendale. 2012. The LunchTable: A Multi-user, Multi-display System for Information Sharing in Casual Group Interactions. In Proceedings of the 2012 International Symposium on Pervasive Displays (PerDis '12), 18:1-18:6. https://doi.org/10.1145/2307798.2307816

30. Sharoda A. Paul and Madhu C. Reddy. 2010. Understanding Together: Sensemaking in Collaborative Information Seeking. In Proceedings of the 2010 ACM Conference on Computer Supported Cooperative Work (CSCW' 10), 321-330. https://doi.org/10.1145/1718918.1718976

31. Peter Pirolli and Stuart Card. 2005. The sensemaking process and leverage points for analyst technology as identified through cognitive task analysis. In Proceedings of international conference on intelligence analysis (Int. Conference on Intelligent Analysis), 2-4. Retrieved July 19, 2015 from

http://vadl.cc.gatech.edu/documents/2_card-sensemaking.pdf

32. Thomas Plank, Hans-Christian Jetter, Roman Rädle, Clemens N. Klokmose, Thomas Luger, and Harald Reiterer. 2017. Is Two Enough?: ! Studying Benefits, Barriers, and Biases of Multi-Tablet Use for Collaborative Visualization. In Proceedings of the 2017 CHI Conference on Human Factors in Computing Systems (CHI '17), 4548-4560. https://doi.org/10.1145/3025453.3025537

33. Christopher Plaue and John Stasko. 2009. Presence \& Placement: Exploring the Benefits of Multiple Shared Displays on an Intellective Sensemaking Task. In Proceedings of the ACM 2009 International Conference on Supporting Group Work (GROUP '09), 179-188. https://doi.org/10.1145/1531674.1531701

34. Roman Rädle, Hans-Christian Jetter, Nicolai Marquardt, Harald Reiterer, and Yvonne Rogers. 2014. HuddleLamp: Spatially-Aware Mobile Displays for Ad-hoc Around-the-Table Collaboration. In Proceedings of the Ninth ACM International Conference on Interactive Tabletops and Surfaces (ITS '14), 45-54. https://doi.org/10.1145/2669485.2669500

35. Daniel M. Russell, Mark J. Stefik, Peter Pirolli, and Stuart K. Card. 1993. The cost structure of sensemaking. In Proceedings of the INTERACT'93 and CHI'93 conference on Human factors in computing systems (CHI '93), 269-276. Retrieved July 19, 2015 from http://dl.acm.org/citation.cfm?id=169209

36. Mario Schreiner, Roman Rädle, Hans-Christian Jetter, and Harald Reiterer. 2015. Connichiwa: A Framework for Cross-Device Web Applications. In Proceedings of 
the 33rd Annual ACM Conference Extended Abstracts on Human Factors in Computing Systems (CHI EA '15), 2163-2168. https://doi.org/10.1145/2702613.2732909

37. Stacey D. Scott, Sheelagh Carpendale, and Kori Inkpen. 2004. Territoriality in collaborative tabletop workspaces. In Proceedings of the 2004 ACM conference on Computer supported cooperative work (CSCW '04) (CSCW '04).

38. Julian Seifert, Adalberto Simeone, Dominik Schmidt, Paul Holleis, Christian Reinartz, Matthias Wagner, Hans Gellersen, and Enrico Rukzio. 2012. MobiSurf: Improving Co-located Collaboration Through Integrating Mobile Devices and Interactive Surfaces. In Proceedings of the 2012 ACM International Conference on Interactive Tabletops and Surfaces (ITS '12), 51-60. https://doi.org/10.1145/2396636.2396644

39. Dennis D. Stewart and Garold Stasser. 1998. The sampling of critical, unshared information in decisionmaking groups: the role of an informed minority. European Journal of Social Psychology 28, 1: 95-113. https://doi.org/10.1002/(SICI)10990992(199801/02)28:1<95::AID-EJSP847>3.0.CO;2-0

40. Anthony Tang, Melanie Tory, Barry Po, Petra Neumann, and Sheelagh Carpendale. 2006. Collaborative coupling over tabletop displays. In Proceedings of the SIGCHI conference on Human Factors in computing systems (CHI '06), 1181-1190. Retrieved January 15, 2015 from http://dl.acm.org/citation.cfm?id=1124950

41. Sarah Tausch, Doris Hausen, Ismail Kosan, Andrey Raltchev, and Heinrich Hussmann. 2014. Groupgarden: Supporting Brainstorming Through a Metaphorical Group Mirror on Table or Wall. In Proceedings of the 8th Nordic Conference on Human-Computer Interaction: Fun, Fast, Foundational (NordiCHI '14), 541550. https://doi.org/10.1145/2639189.2639215

42. Jo Vermeulen, Kris Luyten, Elise van den Hoven, and Karin Coninx. 2013. Crossing the Bridge over Norman's Gulf of Execution: Revealing Feedforward's True Identity. In Proceedings of the SIGCHI Conference on Human Factors in Computing Systems (CHI '13), 1931-1940. https://doi.org/10.1145/2470654.2466255

43. Katherine Vogt, Lauren Bradel, Christopher Andrews, Chris North, Alex Endert, and Duke Hutchings. 2011. Co-located Collaborative Sensemaking on a Large High-Resolution Display with Multiple Input Devices.
In Human-Computer Interaction - INTERACT 2011, Pedro Campos, Nicholas Graham, Joaquim Jorge, Nuno Nunes, Philippe Palanque and Marco Winckler (eds.). Springer Berlin Heidelberg, 589-604. Retrieved September 13, 2016 from http://link.springer.com/chapter/10.1007/978-3-64223771-3_44

44. James R. Wallace, Stacey D. Scott, Eugene Lai, and Deon Jajalla. 2011. Investigating the Role of a Large, Shared Display in Multi-Display Environments. Computer Supported Cooperative Work (CSCW) 20, 6: 529. https://doi.org/10.1007/s10606-011-9149-8

45. James R. Wallace, Stacey D. Scott, and Carolyn G. MacGregor. 2013. Collaborative Sensemaking on a Digital Tabletop and Personal Tablets: Prioritization, Comparisons, and Tableaux. In Proceedings of the SIGCHI Conference on Human Factors in Computing Systems (CHI '13), 3345-3354. https://doi.org/10.1145/2470654.2466458

46. Glen Whyte. 1991. Decision failures: why they occur and how to prevent them. The Executive 5, 3: 23-31. https://doi.org/10.5465/AME.1991.4274458

47. Wesley Willett, Jeffrey Heer, Joseph Hellerstein, and Maneesh Agrawala. 2011. CommentSpace: Structured Support for Collaborative Visual Analysis. In Proceedings of the SIGCHI Conference on Human Factors in Computing Systems (CHI '11), 3131-3140. https://doi.org/10.1145/1978942.1979407

48. Pawel Wozniak, Nitesh Goyal, Przemysllaw Kucharski, Lars Lischke, Sven Mayer, and Morten Fjeld. 2016. RAMPARTS: Supporting Sensemaking with Spatially-Aware Mobile Interactions. In Proceedings of the 2016 CHI Conference on Human Factors in Computing Systems (CHI '16), 2447-2460. https://doi.org/10.1145/2858036.2858491

49. Johannes Zagermann, Ulrike Pfeil, Roman Rädle, Hans-Christian Jetter, Clemens Klokmose, and Harald Reiterer. 2016. When Tablets Meet Tabletops: The Effect of Tabletop Size on Around-the-Table Collaboration with Personal Tablets. In Proceedings of the 2016 CHI Conference on Human Factors in Computing Systems (CHI '16), 5470-5481. https://doi.org/10.1145/2858036.2858224

50. The Top 10 Things to Do in London 2016 - TripAdvisor. Retrieved September 19, 2016 from https://www.tripadvisor.co.uk/Attractions-g186338Activities-London_England.html 